\title{
WEAKLY ALMOST PERIODIC FUNCTIONS AND THIN SETS IN DISCRETE GROUPS
}

\author{
CHING CHOU
}

\begin{abstract}
A subset $E$ of an infinite discrete group $G$ is called (i) an $R_{W}$-set if any bounded function on $G$ supported by $E$ is weakly almost periodic, (ii) a weak $p$-Sidon set $(1 \leq p<2)$ if on $l^{1}(E)$ the $l^{p}$-norm is bounded by a constant times the maximal $C^{*}$-norm of $l^{1}(G)$, (iii) a $T$-set if $x E \cap E$ and $E x \cap E$ are finite whenever $x \neq e$, and (iv) an $F T$-set if it is a finite union of $T$-sets. In this paper, we study relationships among these four classes of thin sets. We show, among other results, that (a) every infinite group $G$ contains an $R_{W}$-set which is not an $F T$-set; (b) countable weak $p$-Sidon sets, $1 \leq p<4 / 3$ are $F T$-sets.
\end{abstract}

\section{INTRODUCTION}

Let $G$ be an infinite discrete group, $\operatorname{WAP}(G)$ the algebra of weakly almost periodic (w.a.p.) functions on $G$. A subset $E$ of $G$ is called an $R_{W}$-set if every function in $l^{\infty}(G)$ which vanishes off $E$ is w.a.p.; $E$ is called a $T$-set if $E \cap x E$ and $E \cap E x$ are finite whenever $x \in G, x \neq e$, the identity of $G$. It was first proved by W. Rudin [17] that $T$-sets and hence finite unions of $T$-sets are $R_{W^{-}}$ sets. However, they seem to constitute the only known $R_{W}$-sets in the literature. In $\S 3$ we show that every infinite group contains an $R_{W}$-set which is not a finite union of $T$-sets. $R_{W}$-sets have already been studied by W. Ruppert [18]. We need the following characterization of $R_{W}$-sets which is similar to a result of his: a subset $E$ of $G$ is an $R_{W}$-set if and only if it does not contain a set of the form $\left\{x_{i} y_{j}: i=1,2, \ldots, 1 \leq j \leq i\right\}$ or $\left\{x_{i} y_{j}: j=1,2, \ldots, 1 \leq i \leq j\right\}$ where $\left\{x_{i}\right\}$ and $\left\{y_{i}\right\}$ are two sequences of distinct elements in $G$.

For $1 \leq p<2$, a subset $E$ of $G$ is called a weak $p$-Sidon set if there is a finite constant $\tau$ such that $\|f\|_{p} \leq \tau\|f\|_{*}$ whenever $f \in l^{1}(E)$ where $\|\cdot\|_{*}$ denotes the maximal $C^{*}$-algebra norm on $l^{1}(G)$. Weak 1 -Sidon sets are called weak Sidon sets in Picardello [15] and, for abelian $G$, weak $p$-Sidon sets are just $p$-Sidon sets as defined in Edwards and Ross [7]. We show that if $1 \leq p<4 / 3$ and $E$ is a weak $p$-Sidon set then $E$ contains no large squares. This generalizes

Received by the editors November 4, 1988.

1980 Mathematics Subject Classification (1985 Revision). Primary 43A46, 43A60; Secondary 43A30, 43A07.

Key words and phrases. Discrete groups, weakly almost periodic functions, infinite triangles, large squares, large $k$-cubes, wide strips, $T$-sets, weak $p$-Sidon sets, $R_{W}$-sets. 
a result in [7] for abelian groups. Déchamps-Gondim [5] proved that countable Sidon sets in abelian groups are finite unions of $T$-sets. We are able to adopt her proof to show in $\S 4$ that if $1 \leq p<4 / 3$ then countable weak $p$-Sidon sets are finite unions of $T$-sets. J. Bourgain [2] showed that Sidon sets, countable or not, in abelian groups are always finite unions of $T$-sets. It does not seem to be known whether his result holds for $p$-Sidon sets if $1<p<4 / 3$.

On the other hand, $T$-sets can be quite large. Indeed, $\S 4$ also contains the following result which improves a result of ours in [4]: every infinite $G$ contains a $T$-set $E$ such that, for each positive integer $k, E$ has a subset $A$ of the form $A=A_{1} \cdots A_{k}=\left\{x_{1} \cdots x_{k}: x_{i} \in A_{i}, i=1, \ldots, k\right\}$ where $\left|A_{i}\right|=k$ and $|A|=k^{k}$. By a result of Johnson and Woodward [12], we then conclude that every infinite abelian group contains a $T$-set which is not a $p$-Sidon set for any $1 \leq p<2$.

Definitions and general results on $R_{W}$-sets and weak $p$-Sidon sets are contained in $\S 2$.

\section{Preliminaries and general Results}

Throughout this paper, $G$ denotes an infinite discrete group, $N$ the set of positive integers, and for a set $A,|A|$ the cardinality of $A$.

Definition 2.1. (a) If $\left\{x_{i}: i \in N\right\}$ and $\left\{y_{j}: j \in N\right\}$ are two sequences in $G$ such that $(i, j) \rightarrow x_{i} y_{j}$ is a one-one mapping from $N^{2}$ into $G$ then $S=\left\{x_{i} y_{i}: i, j \in\right.$ $N\}$ is called an infinite square in $G$ and the sets $\left\{x_{i} y_{j}: i \in N, 1 \leq j \leq i\right\}$ and $\left\{x_{i} y_{j}: j \in N, 1 \leq i \leq j\right\}$ are called infinite triangles.

(b) If $A, A_{i}, i=1, \ldots, k$, are subsets of $G, A=A_{1} \cdots A_{k},\left|A_{i}\right|=n$ and $|A|=n^{k}$ then $A$ is called a $k$-cube of length $n$.

(c) If $C=A B$ or $B A$ where $A$ is infinite, $|B|=n$, and $(a, b) \rightarrow a b$ or $b a$ is a one-one mapping from $A \times B$ to $A B$ or $B A$, then $C$ is called a strip of width $n$.

A subset $E$ in $G$ is said to contain large $k$-cubes if, for any given $n \in N$, $E$ contains a $k$-cube of length $n . E$ is said to contain wide strips if, for any given $n \in N, E$ contains a strip of width $n$. A 2-cube is called a square in [4] and a 1-cube of length $n$ is just a set with $n$ elements. A $k$-cube of length 2 is also called a parallelepiped of dimension $k$; see Hare [11].

Lemma 2.2. (a) If $E=A B$ where $A$ and $B$ are infinite subsets of $G$ then $E$ contains an infinite square.

(b) Suppose that $E \subset G$. If, for each $n \in N$, there exist subsets $A_{1}, \ldots, A_{k}$ of $G$ such that $\left|A_{i}\right|=n, i=1, \ldots, k$, and $A_{1} \cdots A_{k} \subset E$ then $E$ contains large $k$-cubes.

(c) If $E=A B$ where $A, B \subset G, A$ is infinite and $|B|=n$, then there exists an infinite set $A_{1} \subset A$ such that $A_{1} B$ is a strip (of width $n$ ).

Proof. (a) Suppose that we have chosen $A_{n}=\left\{a_{1}, \ldots, a_{n}\right\} \subset A, B_{n}=$ $\left\{b_{1}, \ldots, b_{n}\right\} \subset B$ such that $\left|A_{n} B_{n}\right|=n^{2}$. Choose $a_{n+1} \in A \backslash A_{n} B_{n} B_{n}^{-1}$ and 
then choose $b_{n+1} \in B \backslash A_{n+1}^{-1} A_{n+1} B_{n}$ where $A_{n+1}=\left\{a_{1}, \ldots, a_{n+1}\right\}$. Let $B_{n+1}=$ $\left\{b_{1}, \ldots, b_{n+1}\right\}$. Then $\left|A_{n+1} B_{n+1}\right|=(n+1)^{2}$. Thus, by induction, $E$ contains an infinite square $\left\{a_{i} b_{j}: i, j \in N\right\}$.

(b) For $k=2$, this result was proved in [13, p. 8]. In general, using the method of [13], it is not hard to show, by induction on $k$, that if $B_{i} \subset G$, $\left|B_{i}\right|=n^{2 i-1}+1, i=1, \ldots, k$, then there exist $A_{i} \subset B_{i}$ such that $\left|A_{i}\right|=n$ and $\left|A_{1} \cdots A_{k}\right|=n^{k}$.

We omit the simple proof of $(\mathrm{c})$.

Note that the proof of (a) also shows that if $\left\{a_{i}\right\}$ and $\left\{b_{j}\right\}$ are two sequences of distinct elements in $G$ then $E=\left\{a_{i} b_{j}: i \in N, 1 \leq j \leq n\right\}$ contains an infinite triangle.

As usual, $l^{\infty}(G)$ denotes the space of bounded complex-valued functions on $G$ with sup norm. For $f \in l^{\infty}(G)$ and $x \in G,{ }_{x} f \in l^{\infty}(G)$ is defined by ${ }_{x} f(y)=f(x y), y \in G . f \in l^{\infty}(G)$ is said to be weakly almost periodic (w.a.p.) if the left orbit $O_{L}(f)=\left\{{ }_{x} f: x \in G\right\}$ of $f$ is relatively weakly compact in $l^{\infty}(G)$. WAP $(G)$, the space of w.a.p. functions on $G$, is a translation invariant $C^{*}$-subalgebra of $l^{\infty}(G)$ and, by Ryll-Nardzewski's fixed point theorem [19], it has a unique two-sided invariant mean $m_{G}$. The following result of Grothendieck [10] is the basic tool in our study of w.a.p. functions.

Lemma 2.3 (Grothendieck's criterion). $f \in l^{\infty}(G)$ is w.a.p. if and only if whenever $\left\{x_{i}\right\}$ and $\left\{y_{j}\right\}$ are two sequences in $G$ and $\lim _{i} \lim _{j} f\left(x_{i} y_{j}\right)$ and $\lim _{j} \lim _{i} f\left(x_{i} y_{j}\right)$ exist, then they are equal.

If $E$ is a subset of $G$ and $A$ a subalgebra of $l^{\infty}(G)$ then $l^{\infty}(E)$ is said to reside in $A$, or, in short, $E$ is an $R_{A}$-set, if whenever $f \in l^{\infty}(G)$ and $f$ vanishes off $E$ then $f \in A$. Clearly, the union of two $R_{A}$-sets is an $R_{A}$-set. For convenience, $R_{\mathrm{WAP}(G)}$-sets will be called $R_{W}$-sets. Since $T$-sets are $R_{W}$-sets (see [4, Lemma 3.2]), finite unions of $T$-sets are $R_{W}$-sets.

Proposition 2.4. Let $E$ be a subset of $G$. Then the following two conditions are equivalent:

(1) $E$ is an $R_{W}$-set;

(2) if $\left\{a_{i}: i \in N\right\}$ is a sequence of distinct elements in $G$ then both the sets

$$
\begin{gathered}
A=\left\{x \in G: x a_{i} \text { is eventually in } E\right\}, \\
B=\left\{x \in G: a_{i} x \text { is eventually in } E\right\}
\end{gathered}
$$

are finite.

Proof. (1) $\Rightarrow(2)$. Suppose that $B$ is infinite. Then there exists a sequence of distinct elements $\left\{b_{j}: j \in N\right\}$ in $G$ such that for each $j,\left\{a_{i} b_{j}: i \in N\right\}$ is eventually in $E$. By replacing $\left\{a_{i}\right\}$ and $\left\{b_{j}\right\}$ by subsequences, we may assume that $\left\{a_{i} b_{j}: i, j \in N\right\}$ is an infinite square; see Lemma 2.2(a). Define $f \in l^{\infty}(G)$ by setting $f\left(a_{i} b_{j}\right)=1$ if $a_{i} b_{j} \in E$ and $i \geq j$ and $f(x)=0$ for all 
other $x \in G$. Then

$$
\lim _{i} \lim _{j} f\left(a_{i} b_{j}\right)=0, \quad \lim _{j} \lim _{i} f\left(a_{i} b_{j}\right)=1,
$$

and hence, by Lemma 2.3, $f \notin \operatorname{WAP}(G)$. Since $f$ vanishes off $E$, by definition, $E$ is not an $R_{W}$-set. Similarly, if $A$ is infinite then $E$ is not an $R_{W}$-set.

$(2) \Rightarrow(1)$. Suppose that $(2)$ holds. Since $\operatorname{WAP}(G)$ is a norm closed linear space, to show that $E$ is an $R_{W}$-set, it suffices to show that $\chi_{A} \in \operatorname{WAP}(G)$ for each $A \subset E$. By Lemma 2.3, it suffices to show that whenever $\left\{a_{i}\right\}$ and $\left\{b_{j}\right\}$ are two sequences in $G$ such that

$$
L_{1}=\lim _{j} \lim _{i} \chi_{A}\left(a_{i} b_{j}\right), \quad L_{2}=\lim _{i} \lim _{j} \chi_{A}\left(a_{i} b_{j}\right)
$$

exist then $L_{1}=L_{2}$. It is easy to see that if either $\left\{a_{i}\right\}$ or $\left\{b_{j}\right\}$ is eventually a constant then $L_{1}=L_{2}$. Therefore, we only have to consider the case that $\left\{a_{i}\right\}$ and $\left\{b_{j}\right\}$ are sequences of distinct elements. We claim that in this case $L_{1}=L_{2}=0$. Indeed, if, say, $L_{1}=1$ then there exists $j_{0}$ such that if $j \geq j_{0}$ then $\lim _{i} \chi_{A}\left(a_{i} b_{j}\right)=1$, i.e., for $j \geq j_{0},\left\{a_{i} b_{j}: i \in N\right\}$ is eventually in $E$. Therefore $B$ is infinite, a contradiction.

Remarks. (1) In order to show that weak Sidon sets ae $R_{W}$-sets, we presented the above proposition at the 1982 Summer Meeting of the American Mathematical Society in Toronto; see Abstracts Amer. Math. Soc. 3 (1982), p. 353. Meanwhile, Ruppert has obtained, independently, several characterizations of $R_{W}$-sets in [18]. His condition (ii) in Theorem 7 of [18] is equivalent to our condition (2) above. For the sake of completeness, we include a proof of our proposition here.

(2) As usual, if $\left\{A_{i}\right\}$ is a sequence of sets then $\liminf A_{i}=\bigcup_{n=1}^{\infty}\left(\bigcap_{i=n}^{\infty} A_{i}\right)$. The above proposition states that $E$ is an $R_{W}$-set if and only if $\lim \inf a_{i} E$ and $\lim \inf E a_{i}$ are finite for any sequence $\left\{a_{i}\right\}$ of distinct elements in $G$.

(3) It is not hard to see that the above proposition can be also stated as follows: a subset $E$ of $G$ is an $R_{W}$-set if and only if it does not contain infinite triangles.

Lemma 2.5. If $E$ is an $R_{W}$-set in an infinite group $G$, then $m_{G}\left(\chi_{E}\right)=0$.

Proof. Since $\chi_{E} \in \mathrm{WAP}(G), m_{G}\left(\chi_{E}\right)$ is well defined. By Ryll-Nardzewski's fixed point theorem [19], $m_{G}\left(\chi_{E}\right)=c$ is the unique constant in the closed convex hull of $O_{L}\left(\chi_{E}\right)$. If $\mathrm{c}>0$, then there exists $\sum_{i=1}^{n} \lambda_{i} \chi_{x_{i} E} \in \operatorname{co} O_{L}\left(\chi_{E}\right)$ (the convex hull of $O_{L}\left(\chi_{E}\right)$ ) such that

$$
\left\|\sum_{i=1}^{n} \lambda_{i} \chi_{x_{i} E}-c\right\|_{\infty}<\frac{c}{2} .
$$

This implies that $\bigcup_{i=1}^{n} x_{i} E=G$. Since $E$ is an $R_{W}$-set, so are $x_{i} E, i=$ $1, \ldots, n$. Therefore, $G=\bigcup_{i=1}^{n} x_{i} E$ is also an $R_{W}$-set and hence $\operatorname{WAP}(G)=$ $l^{\infty}(G)$. This contradicts the well-known fact that $\operatorname{WAP}(G) \nsubseteq l^{\infty}(G)$; see $[3$, p. 
68]. (We can also argue as follows: since $G$ clearly contains infinite triangles, by Proposition 2.4, it is not an $R_{W}$-set.)

A group $G$ is said to be amenable if $l^{\infty}(G)$ has a left invariant mean $\mu: \mu \in$ $l^{\infty}(G)^{*},\|\mu\|=1, \mu \geq 0$ and $\mu\left(l_{x} f\right)=\mu(f)$ for all $f \in l^{\infty}(G)$ and $x \in G$. For example, solvable groups are amenable but nonabelian free groups are not amenable; see Pier [14]. If $G$ is amenable, let $\operatorname{LIM}(G)$ be the set of all left invariant means on $G$ and, for $E \subset G$, let $\bar{d}_{l}(E)=\sup \left\{\mu\left(\chi_{E}\right): \mu \in \operatorname{LIM}(G)\right\}$, the left upper density of $E$. For amenable $G$, if $\chi_{E} \in \operatorname{WAP}(G)$ then $m_{G}\left(\chi_{E}\right)=$ $\bar{d}_{l}(E)$. Therefore, by the above lemma, if $\bar{d}_{l}(E)>0$ then $E$ is not an $R_{W}$-set. By Proposition 2.4, we obtain the following.

Corollary 2.6. If $G$ is an infinite amenable group, $E \subset G$ and $\bar{d}_{l}(E)>0$ then $E$ contains infinite triangles.

Remark. Let $\mathbf{Z}$ be the additive group of integers. It is easy to construct a subset $E$ of $\mathbf{Z}$ such that (i) $E$ contains infinite triangles, (ii) $\bar{d}_{l}(E)=0$ and (iii) $E$ does not contain arithmetic progressions of length 3. Note that a celebrated result of E. Szemiredi [20] states that if $E \subset \mathbf{Z}$ and $\bar{d}_{l}(E)>0$ then $E$ contains arbitrarily long arithmetic progressions; see Furstenberg [9] for an ergodic theoretical proof of this result.

Let $C^{*}(G)$ be the completion of $l^{1}(G)$ with respect to the maximal $C^{*}$-norm $\|\cdot\|_{*}:$ for $f \in l^{1}(G)$,

$$
\|f\|_{*}=\sup \{\|\pi(f)\|: \pi \text { a unitary representation of } G\},
$$

where $\pi(f)=\sum\{f(x) \pi(x): x \in G\}$. Then the dual Banach space of $C^{*}(G)$ can be identified with $B(G)$ (the Fourier-Stieltjes algebra of $G$ ) which consists of coefficient functions of unitary representations of $G$. Let $B_{\lambda}(G)$ be the algebra of coefficient functions of unitary representations of $G$ which are weakly contained in the left regular representation $\lambda$. Then $B_{\lambda}(G)$ can be identified with the dual Banach space of $C_{\lambda}^{*}(G)$, the $C^{*}$-algebra generated by $\{\lambda(f): f \in$ $\left.l^{1}(G)\right\}$. See Eymard [8], for definitions and results mentioned in this paragraph.

If $E$ is a subset of $G$ and $f \in l^{1}(E)$ then $f$ will be identified with the function on $G$ which equals $f$ on $E$ and is identically zero off $E$. For $1 \leq$ $p<2$, a subset $E$ of $G$ is called a weak $p$-Sidon ( $p$-Sidon) set if there is a finite constant $\tau$ such that $\|f\|_{p} \leq \tau\|f\|_{* *}\left(\|f\|_{p} \leq \tau\|\lambda(f)\|\right)$ for each $f \in l^{1}(E)$. Note that $p$-Sidon sets are always weak $p$-Sidon and if $G$ is amenable then weak $p$-Sidon sets are $p$-Sidon, since, in this case $\|f\|_{*}=\|\lambda(f)\|, f \in l^{1}(G)$; see [8]. For abelian $G$, a weak $p$-Sidon set is just a $p$-Sidon set as defined by Edwards and Ross [7]. Note also that (weak) 1-Sidon sets are just (weak) Sidon sets as defined by Picardello [15]. Furthermore, a subset $E$ of $G$ is a weak Sidon set (Sidon set) if and only if $B(G) \mid E=l^{\infty}(E) \quad\left(B_{\lambda}(G) \mid E=l^{\infty}(E)\right)$. We showed in [4] that weak Sidon sets do not contain large squares. This result can be strengthened somewhat with a minor change of the proof. 
Proposition 2.7. If $1 \leq p<4 / 3$ and $E$ is a weak $p$-Sidon set in $G$ then $E$ does not contain large squares.

Proof. The proof is similar to that of Proposition 3.4 of [4]. We will give only an outline here. Suppose that $E$ contains large squares. Then for each $n$, choose a square $S=A B$ in $E$ of length $n$, where $A=\left\{a_{1}, \ldots, a_{n}\right\}$ and $B=\left\{b_{1}, \ldots, b_{n}\right\}$. Let $\left(u_{i j}\right)$ be an $n \times n$ unitary matrix with complex entries and with $\left|u_{i j}\right|=1 / \sqrt{n}$. Let $g=\sum_{i, j=1}^{n} u_{i j} \delta_{a_{i} b_{j}}$ where for $t \in G, \delta_{t}$ denotes the function on $G$ which equals 1 at $t$ and zero elsewhere. Then

$$
\|g\|_{p}=\left(\sum_{i, j=1}^{n}\left|u_{i j}\right|^{p}\right)^{1 / p}=n^{2 / p-1 / 2} .
$$

On the other hand, as proved in [4], $\|g\|_{*} \leq n$. Therefore, if $E$ is a weak $p$-Sidon set, then $2 / p-1 / 2 \leq 1$ or $p \geq 4 / 3$, a contradiction.

When $G$ is abelian, the above result is due to Edwards and Ross [7, Corollary 2.7].

Corollary 2.8. For $1 \leq p<4 / 3$, if $E$ is a weak $p$-Sidon set in $G$ then $E$ is an $R_{W}$-set; in particular, $\chi_{E} \in \mathrm{WAP}(G)$.

Proof. By Proposition 2.7, $E$ does not contain large squares and hence it does not contain infinite triangles. By Proposition 2.4, $E$ is an $R_{W}$-set.

Remarks. (1) If $G$ is an infinite abelian group, then it contains an infinite square $S$ such that $S$ is a 4/3-Sidon set; see [7, Corollary 5.5]. By Proposition 2.4, $S$ is not an $R_{W}$-set. Therefore the above result does not hold if $p \geq 4 / 3$.

(2) If the set $E$ in the above corollary is countable, one can actually conclude that $E$ is a finite union of $T$-sets; see $\S 4$.

(3) If $G$ is abelian, a well-known result of Drury [6] states that if $E$ is a Sidon set then $\chi_{E} \in B(G)^{-}$, the uniform closure of $B(G)$. Note that, for every infinite group $G, B(G)^{-}$is properly contained in $\operatorname{WAP}(G)$; see [4].

Lemma 2.9. For $E \subset G$ and $n \in N$, consider the following conditions:

(i) $E$ is a union of $n$-sets;

(ii) $E$ contains no strips of width $n+1$;

(iii) for any finite set $F$ in $G,\{x \in G:|x F \cap E|>n\}$ and $\{x \in G:|F x \cap E|>$ n) are finite.

Then (i) $\Rightarrow$ (ii) and (ii) $\Rightarrow$ (iii).

Proof. (i) $\Rightarrow$ (ii). Let $E=E_{1} \cup \cdots \cup E_{n}$ where $E_{1}, \ldots, E_{n}$ are $T$-sets. Suppose that $E$ contains a strip $C$ of width $n+1$, say $C=B A$ where $B=\left\{b_{1}, \ldots, b_{n+1}\right\}$ and $A=\left\{a_{1}, a_{2}, \ldots\right\}$. By replacing $A=\left\{a_{j}\right\}$ by a subsequence, if necessary, we may assume that, for each $1 \leq i \leq n+1, b_{i} A$ is contained in some $E_{k}, 1 \leq k \leq n$. So there exist $i_{1}, i_{2} \in\{1,2, \ldots, n+1\}$, $i_{1} \neq i_{2}$, such that $b_{i_{1}} A \cup b_{i_{2}} A \subset E_{k_{0}}$ for some $1 \leq k_{0} \leq n$. This contradicts the fact that $E_{k_{0}}$ is a $T$-set. 
(ii) $\Rightarrow$ (iii). Suppose that there exist a finite set $F$ and a sequence of distinct elements $\left\{x_{k}\right\}$ in $G$ such that $\left|x_{k} F \cap E\right|>n$ for all $k$. Then for each $k$ there is a set $F_{k} \subset F$ such that $\left|F_{k}\right|=n+1$ and $x_{k} F_{k} \subset E$. Since $F$ contains only finitely many subsets with cardinality $n+1$, there exists an infinite subset $I$ of $N$ and $F^{\prime} \subset F$ such that, if $k \in I$, then $F_{k}=F^{\prime}$. Then $\left\{x_{k}: k \in I\right\} F^{\prime}$ is a strip of width $n+1$ contained in $E$.

For convenience, we call a set $E$ in $G$ an $F T$-set if it is a finite union of $T$-sets. It is not hard to see that a subset $E$ of $G$ is a $T$-set if and only if given any finite subset $\Delta$ of $G$ there exists a finite subset $F$ of $E$ such that $x, y \in E \backslash F$ and $x \neq y$ imply $x y^{-1}, x^{-1} y \notin \Delta$. Therefore, in the terminology of [13, p. 112], a set $E$ is a $T$-set if and only if it tends to infinity.

\section{Existence of $R_{W}$-SETS Which ARE NOT $F T$-SETS}

As in [4], a subset $E$ of $G$ is said to be relatively dense if there exist finite sets $X$ and $Y$ such that $G=X E Y$. We need the following result of ours in [4].

Lemma 3.1. Let $S$ be a relatively dense subset of $G$ and $F$ a finite subset of $G, e \notin F$. Then there exists a relatively dense subset $E$ of $S$ such that

$$
(x E \cap E) \cup(E x \cap E)=\varnothing
$$

for $x \in F$.

Lemma 3.2. Let $P$ be a relatively dense subset of $G$, say $G=X P Y$ where $X$ and $Y$ are finite. Then for each positive integer $n$ there exists a finite set $E$ such that the set

$$
Q=\left\{x \in G:\left|x E b^{-1} \cap P\right| \geq n \text { for some } b \in Y\right\}
$$

is relatively dense in $G$. In particular, $Q$ is infinite.

Proof. Choose any finite subset $E=\left\{z_{1}, \ldots, z_{k}\right\}$ of $G$ such that $k=|E|=$ $|X||Y| n$. Fix $x \in G$. Then, for each $1 \leq i \leq k, x z_{i}$ can be written as $x z_{i}=a_{i} p_{i} b_{i}$ where $a_{i} \in X, b_{i} \in Y$, and $p_{i} \in P$. For $(a, b) \in X \times Y$, let

$$
I(a, b)=\left\{i: 1 \leq i \leq k, a_{i}=a, b_{i}=b\right\} .
$$

Then $\bigcup\left\{I_{(a, b)}:(a, b) \in X \times Y\right\}=\{1,2, \ldots, k\}$, and hence

$$
k=\sum\left\{\left|I_{(a, b)}\right|:(a, b) \in X \times Y\right\} .
$$

Since $k=|X||Y| n$, there exists $(c, d) \in X \times Y$ such that $\left|I_{(c, d)}\right| \geq n$. If $i \in I_{(c, d)}$ then

$$
c^{-1} x z_{i} d^{-1}=p_{i} \in\left(c^{-1} x E d^{-1}\right) \cap P
$$

and the $p_{i}$ 's, $i \in I_{(c, d)}$, are distinct. Therefore, $c^{-1} x \in Q$. Thus $G=X Q$ and hence $Q$ is relatively dense.

We are now ready to give the main result of this section. 
Theorem 3.3. Let $G$ be an infinite group. Then there exists a subset $D$ of $G$ such that

(a) D is not an FT-set;

(b) $D$ is an $R_{W}$-set.

Proof. Without loss of generality, we may assume that $G$ is countably infinite. Then there exists a sequence of finite symmetric subsets $\left\{F_{n}\right\}$ of $G$ such that

$$
e \in F_{1} \subset F_{2} \subset \ldots, \text { and } G=\bigcup F_{n} .
$$

(A set $B \subset G$ is symmetric if $B=B^{-1}$.) By Lemma 3.1, we can find a sequence of relatively dense subsets $S_{n}$ of $G$ such that $S_{1} \supset S_{2} \supset \cdots$, and

$$
\left(x S_{n} \cap S_{n}\right) \cup\left(S_{n} x \cap S_{n}\right)=\varnothing, \quad \text { if } x \in F_{n} \backslash\{e\} .
$$

For each $n$, choose finite sets $X_{n}$ and $Y_{n}$ such that $X_{n} S_{n} Y_{n}=G$. By Lemma 3.2 , for each $n \in N$, there exists a finite set $E_{n}$ such that

$$
Q_{n}=\left\{x \in G:\left|x E_{n} b^{-1} \cap S_{n}\right| \geq n \text { for some } b \in Y_{n}\right\}
$$

is infinite.

Fix infinite subsets $N_{1}, N_{2}, \ldots$ of $N$ such that $N_{i} \cap N_{j}=\varnothing$, if $i \neq j$, and $N_{1} \cup N_{2} \cup \cdots=N$. Then for each $n \in N$ there is a unique positive integer $\sigma(n)$ such that $n \in N_{\sigma(n)}$.

Choose $t_{1} \in Q_{\sigma(1)}, b_{1} \in Y_{\sigma(1)}$ such that $\left|t_{1} E_{\sigma(1)} b_{1}^{-1} \cap S_{\sigma(1)}\right| \geq \sigma(1)$. Suppose that we have chosen $t_{j}, b_{j}$ in $G, j=1, \ldots, n$, such that the $t_{j}$ 's are distinct, $b_{j} \in Y_{\sigma(j)}$ and if $D_{j}=t_{j} E_{\sigma(j)} b_{j}^{-1} \cap S_{\sigma(j)}$, then $\left|D_{j}\right| \geq \sigma(j)$ and $F_{j} D_{j} F_{j} \cap\left(D_{1} \cup\right.$ $\left.\cdots \cup D_{j-1}\right)=\varnothing$, for $2 \leq j \leq n$. Now since $Q_{\sigma(n+1)}$ is infinite there exists $t_{n+1} \in Q_{\sigma(n+1)}$ such that

$$
t_{n+1} \notin F_{n+1}\left(D_{1} \cup \cdots \cup D_{n}\right) F_{n+1} Y_{\sigma(n+1)} E_{\sigma(n+1)}^{-1} \cup\left\{t_{1}, \ldots, t_{n}\right\} .
$$

Since $t_{n+1} \in Q_{\sigma(n+1)}$, there exists $b_{n+1} \in Y_{\sigma(n+1)}$ such that if $D_{n+1}=t_{n+1}$. $E_{\sigma(n+1)} b_{n+1}^{-1} \cap S_{\sigma(n+1)}$ then $\left|D_{n+1}\right| \geq \sigma(n+1)$. By (3.2),

$$
F_{n+1} D_{n+1} F_{n+1} \cap\left(D_{1} \cup \cdots \cup D_{n}\right)=\varnothing .
$$

Therefore, by induction, we can construct two sequences $\left\{t_{n}\right\}$ and $\left\{b_{n}\right\}$ in $G$ such that the $t_{n}$ 's are distinct, $b_{n} \in Y_{\sigma(n)}$ and if $D_{n}=t_{n} E_{\sigma(n)} b_{n}^{-1} \cap S_{\sigma(n)}$ then

$$
\begin{gathered}
\left|D_{n}\right|=\left|D_{n} \cap S_{\sigma(n)}\right| \geq \sigma(n) ; \\
F_{n} D_{n} F_{n} \cap\left(D_{1} \cup \cdots \cup D_{n-1}\right)=\varnothing, \quad n \geq 2 .
\end{gathered}
$$

Since $D_{n} \subset S_{\sigma(n)},(3.1)$ implies that

$$
\text { if } x \in F_{\sigma(n)} \backslash\{e\} \text {, then }\left(x D_{n} \cap D_{n}\right) \cup\left(D_{n} x \cap D_{n}\right)=\varnothing \text {. }
$$

Also note that, as a consequence of (3.4), we have

$$
\text { if } x \in F_{n}, m \geq n \text { and } l \neq m \text {, then }\left(x D_{m} \cap D_{l}\right) \cup\left(D_{m} x \cap D_{l}\right)=\varnothing \text {. }
$$


We claim that $D=\bigcup_{n=1}^{\infty} D_{n}$ satisfies conditions (a) and (b) in the statement of the theorem.

We will first prove that $D$ satisfies (a). Fix $k \in N$. Note that if $n \in N_{k}$ then $b_{n} \in Y_{k}$. Since $N_{k}$ is infinite and $Y_{k}$ is finite, there exist an infinite subset $I_{k}$ of $N_{k}$ and an element $b \in Y_{k}$ such that if $n \in I_{k}$ then $b_{n}=b$. Let $F_{k}=E_{k} b^{-1}$. Then, for $n \in I_{k}$,

$$
D \cap t_{n} F_{k}=D \cap t_{n} E_{k} b_{n}^{-1} \supset D_{n}
$$

and hence $\left|D \cap t_{n} F_{k}\right| \geq\left|D_{n}\right| \geq k$. Since $I_{k}$ is infinite and $\left\{t_{n}: n \in N\right\}$ is a sequence of distinct elements in $G$, by (i) $\Rightarrow$ (iii) of Lemma 2.9, we conclude that $D$ is not a union of $k-1 \quad T$-sets. Since $k \in N$ is arbitrary, $D$ is not an $F T$-set, as claimed.

It remains to show that $D$ satisfies (b). To this end, first let $x \in F_{k} \backslash\{e\}$, $k \geq 2$, be fixed. Then, by (3.6),

$$
x D \cap D \subset\left\{\left(x D_{1} \cup \cdots \cup x D_{k-1}\right) \cap D\right\} \cup\left\{\bigcup_{m \geq k}\left(x D_{m} \cap D_{m}\right)\right\} .
$$

Note that if $m \notin N_{1} \cup \cdots \cup N_{k-1}$, i.e., $\sigma(m) \geq k$, then $x \in F_{k} \subset F_{\sigma(m)}$, and hence, by (3.5), $x D_{m} \cap D_{m}=\varnothing$. Therefore, (3.7) implies that

$$
x D \cap D \subset F_{x} \cup\left(\bigcup\left\{x D_{m} \cap D_{m}: m \in N_{1} \cup \cdots \cup N_{k-1}\right\}\right),
$$

where $F_{x}=\left(x D_{1} \cup \cdots \cup x D_{k-1}\right) \cap D$ is a finite set. Now assume that $\left\{a_{i}: i \in N\right\}$ is a sequence of distinct elements in $D, x \in F_{k} \backslash\{e\}$ and $\left\{x a_{1}, x a_{2}, \ldots\right\}$ is eventually contained in $D$; in other words $\left\{a_{i}\right\}$ is eventually contained in $x^{-1} D \cap D$. Since $x^{-1} \in F_{k} \backslash\{e\}$, by (3.8), $\left\{a_{i}\right\}$ is eventually contained in $\bigcup\left\{D_{m}: m \in N_{1} \cup \cdots \cup N_{k-1}\right\}$. Similarly, we can prove that if $x \in F_{k} \backslash\{e\}$ and $\left\{a_{i}\right\}$ and $\left\{a_{i} x\right\}$ are both eventually contained in $D$ then $\left\{a_{i}\right\}$ is eventually contained in $\left\{D_{m}: m \in N_{1} \cup \cdots \cup N_{k-1}\right\}$.

Suppose that $D$ is not an $R_{W}$-set. Then, by Proposition 2.4, there exist two sequences $\left\{a_{i}\right\},\left\{y_{j}\right\}$ of distinct elements in $G$ such that either

(I) $\left\{y_{j} a_{i}: i \in N\right\}$ is eventually contained in $D$ for each $j$, or

(II) $\left\{a_{i} y_{j}: i \in N\right\}$ is eventually contained in $D$ for each $j$.

By symmetry, we only have to consider case (I). By renaming the two given sequences, we may also assume that $y_{1}=e$. Then, as demonstrated in the above paragraph, $\left\{a_{i}\right\}$ is eventually contained in $\bigcup\left\{D_{m}: m \in N_{1} \cup \cdots \cup N_{k-1}\right\}$ for some fixed $k \geq 2$. By taking a subsequence, if needed, we may assume that $\left\{a_{i}\right\}$ is contained in $\bigcup\left\{D_{m}: m \in N_{k_{0}}\right\}$ for some fixed $k_{0}$. Assume that $a_{i} \in D_{m_{i}}$ where $m_{i} \in N_{k_{0}}$. We may further assume that the $m_{i}$ 's are distinct. For a fixed $j, j \neq 1$, since $\left\{a_{i}\right\}$ is eventually contained in $D \cap y_{j}^{-1} D$, by (3.7), $a_{i} \in y_{j}^{-1} D_{m_{i}} \cap D_{m_{i}}$ when $i$ is sufficiently large. Thus, for each $l \in N$ there exists an $i$ such that

$$
\left\{a_{i}=y_{1} a_{i}, y_{2} a_{i}, \ldots, y_{l} a_{i}\right\} \subset D_{m_{i}} .
$$


This is impossible, since $\left|D_{m_{i}}\right| \leq\left|E_{k_{0}}\right|$ for each $i$. Therefore, $D$ is an $R_{W}$-set as claimed.

Remarks. (1) If $G$ is an abelian group then the above proof can be simplified somewhat. Our result seems to be new even for $G=\mathbf{Z}$, the additive group of integers. However, for $\mathbf{Z}$ the set $D$ can be constructed more explicitly as follows.

Write $N$ as a disjoint union of infinite sets $N_{k}, k=1,2, \ldots$, and define $\sigma(n)$ as before. Define blocks of consecutive positive integers $C_{n}, n=$ $1,2, \ldots$, inductively so that

$$
\begin{gathered}
\min C_{n+1}>\max C_{n}+n, \\
\left|C_{n}\right|=(\sigma(n)+1)^{2} .
\end{gathered}
$$

Assume that $C_{n}=\left\{t_{n}, t_{n}+1, \ldots, t_{n}+(\sigma(n)+1)^{2}-1\right\}$. Let

$$
J_{n}=\left\{t_{n}, t_{n}+(\sigma(n)+1), t_{n}+2(\sigma(n)+1), \ldots, t_{n}+\sigma(n)(\sigma(n)+1)\right\}
$$

and $D=\bigcup_{n=1}^{\infty} J_{n}$. Then $D$ is an $R_{W}$-set but is not an $F T$-set.

(2) Let $G^{w}$ be the weakly almost periodic compactification of the discrete group $G$. We can consider $G$ as a subset of $G^{w}$. Then the multiplication on $G$ can be extended to $G^{w}$ which makes $G^{w}$ a semigroup with separately continuous multiplication; cf. [3]. In particular, $G$ acts on the compact space $G^{w}$ by left multiplication. From the definition of $R_{W}$-sets, it is easy to see that a subset $E$ of $G$ is an $R_{W}$-set if and only if $\chi_{E}$ is w.a.p. and $E^{-}$(the closure of $E$ in $G^{w}$ ) is the Stone-Čech compactification of $E$; see Ruppert [18]. $\omega \in G^{w} \backslash G$ is said to be strongly $G$-discrete if there is a neighborhood $U$ of $\omega$ in $G^{w} \backslash G$ such that $x U \cap y U=\varnothing$ if $x, y \in G, x \neq y$. Note that if $E$ is an $F T$-set and $\omega \in E^{-} \backslash G$ then $\omega$ is strongly $G$-discrete. On the other hand, if $D$ is the $R_{W}$-set constructed in Theorem 3.3 , then $D^{-}$is homeomorphic to $\beta D$ and there exists $\omega \in D^{-} \backslash G$ such that $\omega$ is not strongly $G$-discrete.

(3) The set $D$ constructed in Theorem 3.3 contains large squares, since it contains wide strips. Therefore the $R_{W}$-set $D$ is not a weak Sidon set. Hence it implies the known result that $B(G)^{-}$is properly contained in $\operatorname{WAP}(G)$ for every infinite group $G$; see [4].

\section{Further RESUlts ON $F T$-SETS}

Déchamps-Gondim proved in [5] that countable Sidon sets in abelian groups are $F T$-sets. She has actually obtained the following result in her proof: if $E$ is a countable subset of an abelian group $G$ and if $E$ does not contain wide strips then $E$ is an $F T$-set. Her proof, with some minor modifications, also works for nonabelian groups.

Theorem 4.1. A countable subset $E$ of a group $G$ is an FT-set if and only if it does not contain wide strips. 
Proof. The "only if" part of the theorem is true no matter whether $E$ is countable or not; see (i) $\Rightarrow$ (ii) in Lemma 2.9. We will now outline the proof of the "if" part in four steps. Assume that $E$ is a countable subset of $G$ which does not contain strips of width $n+1$.

(I) Given any finite set $\Delta$ in $G$, there exists a finite subset $F$ of $E$ such that

$$
|x \Delta \cap(E \backslash F)| \leq n, \quad x \in G .
$$

Indeed, by (ii) $\Rightarrow$ (iii) in Lemma 2.9 , the set $F^{\prime}=\{x \in G:|x \Delta \cap E|>n\}$ is finite. Let $F=F^{\prime} \Delta$. Then (4.1) holds. See also Lemme 6.1 of [5] and Lemma 8.8 of [13].

(II) Given any finite set $\Delta \subset G, E$ can be written as a disjoint union $E=$ $F \cup\left(\bigcup_{i \in I} F_{i}\right)$ where $F$ is finite, for $i \in I,\left|F_{i}\right| \leq n$, and $F_{i}^{-1} F_{j} \cap \Delta=\varnothing$ if $i \neq j$.

This is Lemme 6.2 of [5]; see also Corollary 8.10 of [13]. We include an outline of its proof here. We may assume that $\Delta$ is symmetric and $e \in \Delta$. By (I) there exists a finite set $F$ such that

$$
\left|x \Delta^{n} \cap(E \backslash F)\right| \leq n
$$

for all $x \in G$. A finite set of the form

$$
\left\{x, x t_{1}, x t_{1} t_{2}, \ldots, x t_{1} \cdots t_{k-1}=y\right\}
$$

is called a $\Delta$-chain in $E \backslash F$ if it is a subset of $E \backslash F$ and $t_{1}, \ldots, t_{k-1} \in \Delta$. For $x, y \in E \backslash F$, we define $x \sim y$ if there is a $\Delta$-chain from $x$ to $y$. Then $\sim$ is an equivalence relation. Note that if $x \sim y, x \neq y$, then $x$ and $y$ can be linked by a $\Delta$-chain $x_{1}=x, x_{2}=x t_{1}, \ldots, x_{k}=x t_{1} \cdots t_{k-1}=y$ such that $x_{1}, \ldots, x_{k}$ are distinct. By (4.2), $k \leq n$. Therefore if $X$ is an equivalence class and $x_{0} \in X$ then any element of $X$ is of the form $x_{0} t$ for some $t \in \Delta^{n}$. By (4.2) again, $|X| \leq n$. Clearly, if $x$ and $y$ are in different equivalence classes then $x^{-1} y \notin \Delta$. Thus the $F_{i}$ 's in (II) can be taken to be the equivalence classes of $\sim$.

(III) There exist $E_{i}, i=1, \ldots, n$, such that $E=E_{1} \cup \cdots \cup E_{n}$ and each $E_{i}$ satisfies the condition that $E_{i} \cap x E_{i}$ is finite if $x \in G, x \neq e$.

Follow the proof of Theorem 9.1 of [13]. But, unlike the proof there, the set $E$ is not assumed to be symmetric and we apply (II) instead of Lemma 8.9 of [13]. Note that the countability of $E$ is needed in the proof of (III).

(IV) $E$ is an $F T$-set.

Write $E=\bigcup_{i=1}^{n} E_{i}$ as in (III). By symmetry, each $E_{i}$ can be written as $E_{i}=\bigcup_{j=1}^{n} E_{i j}$ where, for each $(i, j), E_{i j} x \cap E_{i j}$ is finite whenever $x \neq e$. Therefore, $E=\bigcup_{i, j=1}^{n} E_{i j}$ and each $E_{i j}$ is a $T$-set.

Corollary 4.2. Assume that $E$ is a countable weak p-Sidon set in a group $G$ where $1 \leq p<4 / 3$. Then $E$ is an FT-set.

Proof. By Proposition 2.7, $E$ does not contain large squares and hence does not contain wide strips. By the above theorem, $E$ is an $F T$-set. 
Remarks. (1) As mentioned in $\S 2$, if $p \geq 4 / 3$ then the above corollary is not true.

(2) We do not know whether Theorem 4.1 or Corollary 4.2 holds for uncountable sets. Using completely different arguments, Bourgain [2, Corollaire 3.5] proved that Sidon sets in abelian groups are always $F T$-sets. However, his proof does not carry over to the case of $p$-Sidon sets if $p>1$. A subset $E$ of an abelian group $G$ is said to be exactly $p$-Sidon if $E$ is $p$-Sidon but is not $q$-Sidon for any $q<p$. Blei [1] proved that for any $p, 1<p<2$, and for any infinite abelian group $G$, there exists a countable subset $E$ of $G$ such that $E$ is exactly $p$-Sidon. If $p<4 / 3$, by Corollary 4.2 , his countable $p$-Sidon set is an $F T$-set.

We prove in [4] that, given any infinite group $G$, there exists a $T$-set $E$ in $G$ which contains large squares. This is the key step to show that $B(G)^{-} \nsubseteq$ $\operatorname{WAP}(G)$ for any infinite group $G$; see [4]. (For abelian $G$ this result is due to Rudin [17] and Ramirez [16].) It turns out that a $T$-set can even contain large $k$-cubes for any given $k$. To prove this we need the following refinement of Lemma 3.6 of [4].

Lemma 4.3. There is a function $\alpha: N \times N \rightarrow N$ such that whenever $A=A_{1} \cdots A_{k}$ is a $k$-cube of length $\alpha(k, n)$ and is contained in the union of two subsets $E_{1}$ and $E_{2}$ of a group $G$ then there exist subsets $B_{i}$ of $A_{i}$, such that $\left|B_{i}\right|=n$, $i=1, \ldots, k$, and $B=B_{1} \cdots B_{k}$ is contained in either $E_{1}$ or $E_{2}$.

Proof. Let $\alpha(1, n)=2 n$. For $k \geq 2$, define $\alpha(k, n)$ inductively, by setting

$$
\alpha(k, n)=2 n\left(\begin{array}{c}
\alpha(k-1, n) \\
n
\end{array}\right)^{k-1}
$$

(For integers $m, n, 0 \leq m \leq n,\left(\begin{array}{c}n \\ m\end{array}\right)=\frac{n !}{m !(n-m) !}$. ) Then $\alpha$ is the function we want. We will prove this by induction on $k$.

A 1-cube of length $n$ is just a finite set with $n$ elements. If $A \subset E_{1} \cup E_{2}$, $|A|=2 n=\alpha(1, n)$ then clearly there is a subset $B$ of $A$ such that $|B|=n$ and $B$ is contained in either $E_{1}$ or $E_{2}$. Suppose that our result holds for $k-1$. Let $A=A_{1} \cdots A_{k}$ be a $k$-cube of length $\alpha(k, n)$ and $A \subset E_{1} \cup E_{2}$. Choose subsets $A_{i}^{\prime}$ of $A_{i}, i=1, \ldots, k-1$, such that $\left|A_{i}^{\prime}\right|=\alpha(k-1, n)$. For each $y \in A_{k}$,

$$
A^{\prime}=A_{1}^{\prime} \cdots A_{k-1}^{\prime} \subset E_{1} y^{-1} \cup E_{2} y^{-1} .
$$

Therefore, by inductive assumption, for each $y \in A_{k}$, there exists a $(k-1)$-cube $K(y)=A_{1}(y) \cdots A_{k-1}(y)$ of length $n$ where $A_{i}(y) \subset A_{i}^{\prime}$ and $K(y)$ is contained in either $E_{1} y^{-1}$ or $E_{2} y^{-1}$, or equivalently, $K(y) y$ is contained in either $E_{1}$ or $E_{2}$. Clearly, there exists a set $A_{k}^{\prime} \subset A_{k}$ such that $\left|A_{k}^{\prime}\right|=(1 / 2) \alpha(k, n)$ and either (i) $K(y) y \subset E_{1}$ for all $y \in A_{k}^{\prime}$ or (ii) $K(y) y \subset E_{2}$ for all $y \in A_{k}^{\prime}$. Suppose that (i) holds. Let $\left\{C_{1}, \ldots, C_{l}\right\}$ be the collection of subsets of $A^{\prime}$ of the form $A_{1}^{\prime \prime} \cdots A_{k-1}^{\prime \prime}$ where $A_{i}^{\prime \prime} \subset A_{i}^{\prime}$ and $\left|A_{i}^{\prime \prime}\right|=n, i=1, \ldots, k-1$. Note 
that $l=\left(\begin{array}{c}\alpha(k-1, n) \\ n\end{array}\right)^{k-1}$. Let

$$
D_{i}=\left\{y \in A_{k}^{\prime}: K(y)=C_{i}\right\}, \quad i=1, \ldots, l .
$$

Then $\bigcup_{i=1}^{l} D_{i}=A_{k}^{\prime}$. Therefore, there exists some $i_{0}$ such that $\left|D_{i_{0}}\right| \geq n$; otherwise,

$$
n l=\frac{1}{2} \alpha(k, n)=\left|A_{k}^{\prime}\right|<n l, \quad \text { a contradiction } .
$$

Choose $A_{k}^{\prime \prime}=\left\{y_{1}, \ldots, y_{n}\right\} \subset D_{i_{0}}$ and write $C_{i_{0}}=A_{1}^{\prime \prime} \cdots A_{k-1}^{\prime \prime}$. Then $A_{1}^{\prime \prime} \cdots A_{k}^{\prime \prime}$ $\subset E_{1}$. This completes the proof of the lemma.

The above lemma implies that every relatively dense subset of an infinite group contains large $k$-cubes for each $k \in N$; see [4, p. 146]. As a consequence, we can follow the proof of Proposition 3.10 of [4] to obtain the following.

Theorem 4.4. Let $G$ be an infinite group. Then for each $n \in N$ there exists an $n$-cube $K_{n}$ of length $n$ in $G$ such that $E=\bigcup_{n=1}^{\infty} K_{n}$ is a T-set.

Johnson and Woodward [12] proved that if a subset $E$ of an abelian group contains large $k$-cubes then it is not a $p$-Sidon set for any $p<2 k /(k+1)$. Therefore Theorem 4.4 has the following consequence.

Corollary 4.5. Let $G$ be an infinite abelian group. Then there exists a $T$-set $E$ in $G$ such that $E$ is not a $p$-Sidon set for any $1 \leq p<2$.

We do not know whether the above corollary holds for general infinite groups.

\section{REFERENCES}

1. R. C. Blei, Combinatorial dimension and certain norms in harmonic analysis, Amer. J. Math 106 (1984), 847-887.

2. J. Bourgain, Propriétés de décomposition pour les ensembles de Sidon, Bull. Soc. Math. France 111 (1983), 421-428.

3. R. B. Burckel, Weakly almost periodic functions on semigroups, Gordon and Breach, New York, 1971.

4. C. Chou, Weakly almost periodic functions and Fourier-Stieltjes algebras of locally compact groups, Trans. Amer. Math. Soc. 274 (1982), 141-157.

5. M. Déchamps-Gondim, Ensembles de Sidon topologiques, Ann. Inst. Fourier (Grenoble) 22 (1972), 51-79.

6. S. W. Drury, Sur les ensembles de Sidon, C. R. Acad. Sci. Paris Sér. I Math. 271 (1970), 162-163.

7. R. E. Edwards and K. A. Ross, p-Sidon sets, J. Funct. Anal. 15 (1974), 404-427.

8. P. Eymard, L'algèbre de Fourier d'un groupe localement compact, Bull. Soc. Math. France 92 (19.64), 181-236.

9. H. Furstenberg, Ergodic behavior of diagonal measures and a theorem of Szemerédi on arithmetic progressions, J. Analyse Math. 31 (1977), 204-256.

10. A. Grothendieck, Critères de compacité dans les espaces fonctionnels généraux, Amer. J. Math. 74 (1952), 168-186.

11. K. E. Hare, Arithmetic properties of thin sets, Pacific J. Math . 131 (1988), 143-155.

12. G. W. Johnson and G. S. Woodward, On p-Sidon sets, Indiana Univ. Math. J. 24 (19741975), 161-167. 
13. J. M. López and K. A. Ross, Lecture Notes in Pure and Appl. Math.,, Dekker, New York, 1975.

14. J. Pier, Amenable locally compact groups, Wiley-Interscience, New York, 1984.

15. M. A. Picardello, Lacunary sets in discrete noncommutative groups, Bull. Un. Mat. Ital. 8 (1973), 494-508.

16. D. Ramirez, Weakly almost periodic functions and Fourier-Stieltjes transforms, Proc. Amer. Math. Soc. 19 (1968), 1087-1088.

17. W. Rudin, Weak almost periodic functions and Fourier-Stieltjes transforms, Duke Math. J. 26 (1959), 215-220.

18. W. A. F. Ruppert, On weakly almost periodic sets, Semigroup Forum 32 (1985), 267-281.

19. C. Ryll-Nardzewski, On fixed points of semigroups of endomorphisms of linear spaces, Proc. Fifth Berkeley Sympos. Math. Statist. and Prob., Vol. II, Part I, Theory of Probability, Univ. of California Press, Berkeley, 1966, pp. 55-61.

20. E. Szemerédi, On sets of integers containing no $k$ elements in arithmetic progression, Acta Arith. 27 (1975), 199-245.

Department of Mathematics, State University of New York at Buffalo, Buffalo, NEW YORK 14214 